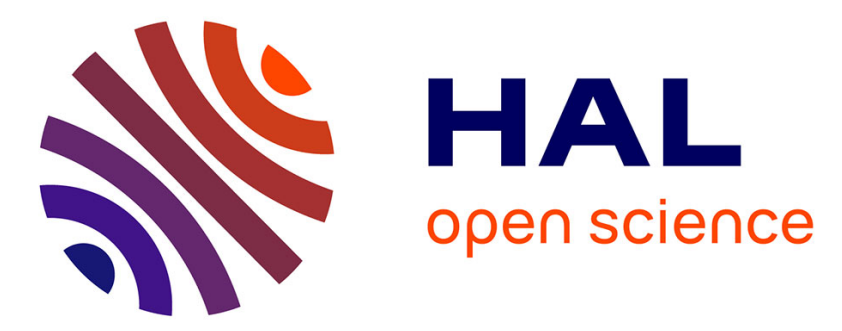

\title{
Inceste, mensonge et filiation une perspective ethnologique de la relation entre frère et sœur en Europe
}

Raymond Jamous

\section{To cite this version:}

Raymond Jamous. Inceste, mensonge et filiation une perspective ethnologique de la relation entre frère et sœur en Europe. Ethnologie française, 2015, Grandir: pouvoirs et périls, 154, pp.745-754. 10.3917/ethn.154.0745 . halshs-01954581

\section{HAL Id: halshs-01954581 https://shs.hal.science/halshs-01954581}

Submitted on 13 Dec 2018

HAL is a multi-disciplinary open access archive for the deposit and dissemination of scientific research documents, whether they are published or not. The documents may come from teaching and research institutions in France or abroad, or from public or private research centers.
L'archive ouverte pluridisciplinaire HAL, est destinée au dépôt et à la diffusion de documents scientifiques de niveau recherche, publiés ou non, émanant des établissements d'enseignement et de recherche français ou étrangers, des laboratoires publics ou privés. 


\title{
inceste, mensonge et filiation une perspective ethnologique de la relation entre frère et sœur en Europe ${ }^{1}$
}

\author{
Raymond Jamous \\ Laboratoire d'ethnologie et de sociologie comparative
}

\section{Résumé en français}

Si en Inde notamment, la relation frère-sœur se définit en rapport à l'alliance du mariage et à la construction rituelle de la filiation, il en va différemment dans la parenté en Europe contemporaine. C'est à partir de trois romans et de trois films considérés comme des récits ethnographiques que nous l'analyserons. Dans ces fictions, c'est l'ambiguité et la tension qui sont au cœur des relations entre ces germains de sexe opposé : dans les romans, c'est un amour fusionnel jusqu'à l'inceste entre frère et sœur, amour stérile qui dévalorise tout autre relation de parenté ; dans les films, c'est la prééminence de la sœur sur le frère, mensonge de la sœur pour éduquer son frère. Il apparaît bien à partir de ces exemples que l'alternative se situe entre l'inceste et la filiation, sans que le mariage soit considéré comme une dimension importante de la construction sociale de la parenté.

Mots- clés: Frère-sœur,Rituel. Roman. Inceste. Parenté. Film.

\section{Résumé en anglais}

Incest, lies and descent. An anthropological perspective on the relationship

\footnotetext{
${ }^{1}$ Remerciements : Je voudrais remercier tout particulièrement ma femme, Marie-Jo Jamous qui a lu et relu les différentes versions de cette article, comme elle l'a fait pour toutes mes publications. Ses nombreuses remarques avisées, ses multiples suggestions m’ont amené à préciser mon propos. Merci aussi à Maryse Bouvet, Charles Malamoud, et Romain Simenel qui ont lu avec attention des versions de ce travail et j'ai bénéficié de leur commentaires qui m'ont permis de peaufiner mon analyse sur de nombreux points.
} 
between brother and sister in Europe

If in India specially the definition of the brother-sister relationship is organized in connection with the marriage alliance and the ritual construction of descent, it is quite different in contemporary European kinship. The analysis of this relationship is based on three novels and three films considered as ethnographic accounts. In these fictions, ambiguity and tension prevail in the relation between opposite sex siblings: in the novels, fusional even incestuous love between brother and sister, sterile love that devaluates any other family relationship ; in the movies, proeminence of the sister over the brother, lies of the sister to educate her brother. From these examples it is clear that the alternative is set between incest and filiation while marriage does not appear as an important dimension

\section{Texte}

D'un point de vue général, la relation entre germains de sexe opposé est la seule qui soit fondée sur l'identité et la différence absolue. Entre frères et sœurs, seule l'identité est marquée ; entre mari et femme, c'est la différence qui est soulignée, de même qu'entre parents et enfants. En revanche, frère et sœur peuvent jouer sur les deux plans et c'est là que se noue toute la complexité de leur rapport : proximité et distance, gémellité et séparation, asymétrie ou identité, attirance et danger, inceste ou mariage etc. On peut alors se demander comment les sociétés se représentent cette germanité de sexe opposé.

La question a été étudiée dans les sociétés non européennes, notamment en Inde, et c'est là que nous prendrons le point de départ et de comparaison. Elle se pose aussi, mais d'une autre manière dans les sociétés occidentales contemporaines que nous étudierons en prenant pour sources quelques romans et fictions cinématographiques ${ }^{1}$.

\section{La relation frère- sœur entre mythe et rituel : l'exemple indien}

Claude Lévi-Strauss a établi sa théorie générale de la parenté sur l'idée que la prohibition de l'inceste consti- tue la face négative de l'ouverture vers l'échange matri- monial : le frère ne pouvant se marier avec sa sœur doit donc la céder à un autre qui doit faire de même, ce qui ouvre le champ de l'alliance matrimoniale [Lévi-Strauss, 1967]. Louis Dumont [1975] a utilisé et formalisé cette approche pour la parenté dravidienne de l'Inde du sud, en soulignant la complémentarité terminologique entre consanguinité et affinité et en montrant la façon dont le mariage asymétrique avec la cousine croisée matrilatérale correspondait à l'un des modèles lévi-straussiens. 
Une autre figure de la double relation (identité et séparation), entre frère et sœur en Inde est pré- sente dans les textes védiques; il s'agit d'une paire de divinités, les jumeaux Yama et Yami. Ici on suivra l'analyse de Charles Malamoud [2002]. Dans ce cadre, l'humanité n'a pas encore été créée et le propos du dialogue entre le frère et la sœur est de savoir si l'inceste est l'acte normal pour faire advenir les humains ou si c'est un acte créateur de chaos. Yami est favorable à l'accouplement alors que son frère est contre. Deux termes s'opposent alors : Jami qui fait référence à la consanguinité, manière de parler de l'identité des deux germains et mithuna qui indique les relations sexuelles entre un homme et une femme et qui, d'une certaine manière, renvoie à une forme d'affinité. Dans ce contexte, on peut dire que Yami veut mettre l'affinité dans la consanguinité alors que Yama s'y refuse et met en valeur la distinction. " Yama et Yami se sont quittés sans avoir accompli l'acte qui devait donner naissance à la race humaine. Mais nul autre récit ne vient relayer celui-ci : l'humanité est le fruit d'un inceste qui n'a pas eu lieu, ou qui, s'il est en fin de compte réalisé, est devenu un évènement sans trace » [ibid.:51]. On retrouve ici une opposition fondamentale en Inde entre consanguinité et affinité avec une vue modifiée de la relation frère-sœur par rapport à la proposition de Claude Lévi-Strauss, puisque cette relation est à l'origine de l'humanité sans qu'il y ait inceste.

Dans mes travaux sur les Meo de l'Inde du nord [Jamous, 1991], la relation entre frère et sœur spécifie encore plus sa centralité dans la parenté. Elle peut se constituer lors de l'enfance, mais elle ne prend toutes ses dimensions qu'après le mariage du frère ou de la sœur. Certes on n'épouse pas sa sœur, et le mariage est supposé les séparer mais c'est là qu'intervient l'importance du rituel. Le frère marié doit demander l'assistance de sa sœur (elle-même mariée) pour être l'officiante principale lors de la naissance et du mariage de ses enfants, de la circoncision de ses fils. Par ailleurs la femme de cet homme doit recevoir des prestations importantes de son frère lorsqu'elle accouche ou marie ses enfants. Ainsi les rites font-ils apparaitre un couple avec la sœur de l'un et le frère de l'autre. La paire frère-sœur apparaît non pas comme celle qui donne la vie mais comme celle qui donne un surcroît de vie rituelle et joue un rôle essentiel pour la mise en place de la descendance. À la génération suivante, la fille du frère prendra la place occupée par sa tante paternelle, et le fils de la sœur celui de son oncle maternel. Ainsi la relation frère-sœur s'inscrit-elle dans la diachronie. Comme on le voit, c'est à travers le mariage et ses conséquences que cette relation prend toute sa valeur et que la filiation, ici patrilinéaire, prend forme. Le 
processus rituel établit donc la suite des générations par cet opérateur central qu'est la paire frère-sœur.

C'est à partir de cette analyse qu'une comparaison me paraît intéressante : comment définit-on la relation frère-sœur par rapport à la filiation et au mariage dans la parenté européenne ? comment la temporalité, c'est-à-dire le présent de la germanité de sexe opposé se positionne-t-elle dans son rapport au passé de l'ascendance et au futur de la descendance ? Pour répondre à ces questions, je travaillerai sur des fictions du $\mathrm{xx}^{\mathrm{e}}$ siècle dans lesquelles cette relation se trouve au premier plan et en les interrogeant comme s'ils étaient des récits ethnographiques sur la parenté. Dans un premier temps, je m'intéresserai à un grand roman, L'Homme sans qualités de Robert Musil et à deux nouvelles Le Sang réservé de Thomas Mann et Confidence africaine de Roger Martin du Gard. Ensuite et plus longuement, trois films retiendront mon attention : Sandra de Luchino Visconti, Mémoires en fuite de François Marthouret, et Bunny Lake a disparu d'Otto Preminger.

\section{Un amour fusionnel sans avenir entre frère- sœur : trois fictions romanesques}

Dans le roman et les deux nouvelles, l'accent est mis de manière différente sur une passion amoureuse fusionnelle entre frère et sœur.

\section{Le Sang réservé}

Dans la nouvelle de Thomas Mann écrite en 1905, ce sont les jumeaux frèresœur Siegmund et Sieglind qui sont au centre de l'histoire. Ni la référence au père ni celle au futur mari de la sœur ne sont valorisées. La représentation d'un opéra de Wagner à laquelle les jumeaux assistent, et qui met en scène la passion entre deux germains de sexe opposé, suscite la leur, alors qu'ils s'identifient aux héros de l'opéra. Après le spectacle, ils traversent une sorte de transe amoureuse. Voici comment Thomas Mann la décrit :

«Son front [celui du frère] brûlait et ses mains étaient moites et glacées. Sieglind s'agenouilla près de lui sur la fourrure et posa sa main sur ses cheveux. Il était à demi soulevé, un bras passé autour du cou de sa sœur et le considérait du même regard dont il avait examiné tout à l'heure sa propre image » [Mann, $1905: 54]$.

Le frère essaya de parler sans que son discours fasse sens : 
«Et rien de tout cela ne paraissait surprendre Sieglind. Elle ne marquait nulle gêne à l'entendre s'exprimer avec tant de passion. Les désordres de ces paroles envelop- paient ses sens, l'attiraient vers la contrée mystérieuse d'où ils étaient nés » [ibid. : 55].

Et voilà comme la soirée se termina :

«Elle baisa ses yeux clos. Il baisa sa gorge sous la den- telle. Ils se baisèrent les mains. Tendrement sensuels, ils s'aimaient pour leurs raffinements et pour leurs parfums. Ils s'abandonnèrent à leur griserie avec une insouciante volupté, s'en droguèrent comme des malades, désespérément, cependant que leurs caresses élevaient en eux un croissant et rapide tumulte qui se termina en un seul sanglot» [ibid. : 55].

\section{L'Homme sans qualités}

Dans le roman de Robert Musil, écrit dans les années 1930-1940, l'auteur consacre une grande partie du tome II, à décrire la relation entre Ulrich et sa sœur Agathe au moment des funérailles de leur père. Plusieurs étapes de leur relation sont ici intéressantes.

Premier moment : le frère et sœur se contemplent, et s'aperçoivent qu'ils sont vêtus de la même façon :

«C'était un grand pyjama de laine moelleuse, une sorte de costume de Pierrot, carrelé de gris et de noir, noué au poignet et aux chevilles comme à la ceinture. Il [Ulrich] l'aimait pour son confort... Mais lorsqu'il pénétra dans la chambre où l'attendait sa sœur, il s'émerveilla de s'être ainsi vêtu. Par une mystérieuse disposition du hasard, il se trouva devant une Pierrot blond, enveloppé de rayures et de carreaux d'un gris et d'un rouille subtils, qui, au premier coup d'œil, paraissait tout semblable à lui. « Je ne savais pas que nous fussions jumeaux » dit Agathe, et son visage s'éclaira de gaieté » [Musil, 1932 : 14$15]$.

Deuxième moment : au cours d'une scène, alors que Agathe s'habille, son frère la soulève, l'enlace et c'est la première occasion de sentir une forme d'unité corporelle :

«Le frère et la sœur se changeaient pour se rendre à une soirée [...] Presque tout l'appareil guerrier qu'une femme utilise en pareille occasion était étalé en pièces détachées sur les dossiers et les moindres surfaces de la chambre, et Agathe venait de se pencher pour enfiler un bas avec toute l'attention que requiert une extrême finesse de la soie. Ulrich était debout dans son dos. Il voyait sa tête, le cou, les épaules et ce dos presque nu ; le corps se courba sur le genou relevé en se déjetant un peu, et la tension du mouvement dessina sur le cou trois plis qui fendirent la peau claire finement, joyeusement, comme trois flèches : la grâce charnelle de ce tableau [...] parut n'avoir plus de cadre et passa 
dans le corps d'Ulrich si directement, si immédiatement qu'il quitta sa place [...] s'approcha sur la pointe des pieds, surprit la jeune femme inclinée et mordit dans l'une des flèches avec une tendre sauvagerie, cependant que d'un bras il l'enlaçait [...] de la main droite il avait saisi le genou et tandis qu'avec le bras gauche il serrait le corps de sa sœur contre le sien, ban- dant les muscles des jambes, il la souleva en l'air. Agathe poussa un cri effrayé [...] Mais quand Agathe surmonta son effroi et qu'elle se sentit non pas voler, mais reposer dans l'air, déliée de toute pesanteur et soumise en lieu et place à la tendre pression d'un mouvement de plus en plus lent, un de ces hasards qui ne sont au pouvoir de per- sonne fit qu'elle se trouva merveilleusement apaisée, ravie même à toutes les agitations de la terre « [ibid. : 465-466] .

Dans ce moment : « tout ce qu'ils faisaient leur paraissait remarquablement libre de toute force, de toute contraintes » [ibid. : 466].

Enfin troisième moment : le frère et la sœur partent sur une île pour vivre leur passion et pour mourir :

« Agathe se laissa aller, à demie évanouie, sur la poitrine d'Ulrich. En cet instant, elle se sentit étreinte par son frère d'une manière si ample, si calme, si pure qu'il n'existait rien de semblable au monde. Leurs corps ne bougeaient pas et n'étaient pas changés : pourtant un bonheur sensuel dont ils n'avaient jamais connu l'équivalent les envahissait [...] Où qu'ils se touchassent, fût-ce aux hanches, aux mains ou dans les cheveux, ils pénétraient l'un dans l'autre. Tous deux furent convaincus, à ce moment-là qu'ils n'étaient plus soumis aux séparations humaines. Ils avaient dépassé le stade du désir qui dépense son énergie en un acte et une brève exaltation ; l'accomplissement ne leur venait pas seulement en certains endroits de leur corps, mais partout, comme un feu ne baisse pas quand un autre feu s'y allume » [ibid. : 835-836].

Après ces moments d'une sorte d'union mystique durant laquelle le frère et la sœur ne font plus qu'un, c'est la séparation entre eux qui finit par s'imposer. Le roman ne dit jamais qu'ils ont des rapports sexuels incestueux mais tout le suggère. Durant cette période au cours de laquelle Agathe et Ulrich vivent leur amour, le texte de Musil minimise toute relation de parenté autre que la relation frère-sœur : ainsi le frère est célibataire ; Agathe devenue veuve rejette le remariage ; la relation au père est minorée comme dans la nouvelle de Thomas Mann, même si les deux protagonistes se retrouvent au moment du décès de ce dernier et entament alors leur relation.

Dans les deux textes, la relation sexuelle n'est jamais explicite, elle est suggérée. Tous deux centrent le récit sur la relation frère-sœur. L'action se déroule au présent et semble dévaloriser la consanguinité comme le mariage, en l'absence de référence à une descendance ou à une parenté plus large. On est à la limite de 
la parenté.

\section{Confidence africaine}

Si la nouvelle de Roger Martin du Gard diffère des textes de Musil et de Mann, elle les rejoint cependant sur le fond. Le narrateur conte les confidences d'un Italien Leandro, libraire dans un pays africain. Dans ce récit, il est question des circonstances qui l'ont amené à avoir avec sa sœur Amalia, pendant quatre ans, une relation incestueuse assumée et vécue à l'abri des regards du monde extérieur. Relégués par leur père (qu'ils n'ai- ment pas) dans une chambre qu'ils partagent, c'est après que Leandro ait eu une relation sexuelle avec une jeune femme dans leur logis que la tension monte entre le frère et la sœur, et c'est au cours d'une bagarre entre eux qu'ils en viendront à avoir une relation incestueuse :

«On était tous les deux en chemise, pressés l'un contre l'autre dans le noir, et on luttait comme deux forcenés. J'avais fini par la soulever. Elle me griffait la nuque. Moi je reniflais cette chair encore chaude au lit [...] D'un coup brusque, j'ai fait plier ses reins et je l'ai renversé sur mon matelas. à ce moment-là, je me suis trouvé pris entre ses deux jambes, entre ses jambes nues, qu'elle a refermées derrière moi. J'ai basculé. Je suis tombé sur elle. Ma colère, je vous avoue qu'il n'en restait pas grand chose [...] juste de quoi exaspérer mon désir. Alors, j'ai recherché ses lèvres, rageusement. Je crois bien que déjà elle me tendait maladroitement les siennes « [Martin du Gard, 1949 : 64-65].

Après quatre ans de relations incestueuses, et avant qu'Amalia n'accepte d'épouser Luzzetti, l'adjoint du père dans la librairie, celui qu'elle appelle le « vieux porc », elle demande à son frère de lui faire un enfant. Prénommé Michele, l'enfant naît chétif, malingre, fragile et ne pourra pas s'épanouir comme les quatre enfants que Amalia aura de son mari. Leandro restera célibataire et vivra dans la maison de son père auprès de la famille de sa sœur, qui a beaucoup grossi, qui « se «bâfre » de bouilli de figues de miel » et ne lui rappelle plus l'Amalia de sa jeunesse. Michele va mourir dans un centre médical veillé par son père biologique. Le récit se conclut ainsi :

«Pour elle [Amalia] - sans qu'elle se soit rendu compte - la mort de Michele, ç'a été un soulagement. Oui. Et au fond, tenez [...] pour moi aussi. Malgré le chagrin que j'ai eu, je suis heureux. Je suis même plus heureux, plus tranquille, qu'avant. Nous sommes tous heureux. Ensemble. C'est ainsi et on n'y peut rien » [ibid. : 86-87]. 
La mort de l'enfant issu de l'inceste, est acceptée comme une délivrance, et même comme allant de soi. À la différence des deux récits précédents, celui-ci ne narre pas un amour passionnel, une transe entre le frère et la sœur. L'inceste est assumé par les deux, semble se dérouler un peu comme étant le fruit du hasard. La naissance de l'enfant et sa mort ne font l'objet d'aucun drame mais semblent aller de soi. La relation incestueuse n'a donc pas conduit à la fondation d'une lignée, contrairement à la «famille ordinaire » de la sœur, tandis que le frère reste célibataire. Cette relation est sans avenir et l'on retrouve ainsi, d'une autre manière, l'idée que frère et sœur incestueux se situent pratiquement hors de la parenté. Dans les trois fictions romanesques, la relation frère-sœur se manifeste à l'âge adulte et joue sur les deux registres de la différence et de l'identité, sur le rapport fusionnel, l'inceste étant vécu parfois comme une transe ou une union mystique.

\section{La prééminence de la sœur : trois fictions cinématographiques}

Dans les trois films dont l'analyse suit, il n'est plus question d'identification entre le frère et la sœur, mais de la façon dont est géré leur conflit, les deux protagonistes se différenciant avec une prédominance de la sœur sur son frère.

Le désir d'inceste et le culte du père : Sandra

Dans ce film de Luchino Visconti de 1965, Sandra (Claudia Cardinale) revient à Volterra, sa ville natale, avec son mari Andrew, pour assister à une cérémonie de donation du jardin familial à la municipalité et à l'inauguration de la statue en mémoire de son père déporté et mort à Auschwitz. Elle y retrouve son frère Gianni (Jean Sorel), jeune écrivain avec lequel elle a entretenu durant leur jeunesse une relation ambiguë et probablement incestueuse.

Tout le propos du film est de situer l'évolution contrastée de leur relation. Le frère ne s'est pas marié et a vécu des aventures sans lendemain. Il a écrit un livre sur l'amour passionnel entre un frère et une sœur, sur le désir incestueux. Le titre du livre Vaghe stelle dell' Orsa est celui du film en italien. Gianni avoue à Sandra à qui il a donné à lire le manuscrit qu'il s'agit bien de leur aventure et que personne ne sera dupe.

Dans le film, Gianni apparaît donc comme un personnage sans ambiguïté qui aime passionnément Sandra et ne cesse de lui demander de partager son amour. Le désir incestueux ne le rebute pas, bien au contraire. A sa sœur qui lui 
reproche de ne pas être honteux de son comportement, il répliquera :

«Pourquoi aurais-je honte ? [...] Pourquoi tu te sens coupable ? Pourquoi nous devons nier la joie et le plaisir que nous avons partagé [...] Tu étais terrifiée à l'idée de t'identifier à moi et tu as voulu t'éloigner de ce passé que nous avons partagé. Tu as été au camp de concentration pour retrouver la trace de notre père et pour rappeler son calvaire. Tu as caché ton amour pour moi sous le masque de ton sacrifice [pour le culte du père]. Tu as persécuté une pauvre femme malade [leur mère]. Tu as renvoyé ton mari qui t'aime comme moi je t'aime. Tu peux penser que je suis cynique et corrompu mais je suis honnête et je suis capable d'un grand geste. J'ai brûlé mon livre pour toi. Aide moi, reste avec moi, ne m'abandonne pas de nouveau. Je t'implore, je ferais n'importe quoi pour toi [...] Si tu m'abandonnes, je me tuerai, je le jure, tu le regretteras ».

Au terme de cette supplique, Sandra lance d'un air sombre : « tu es déjà mort pour moi ». Gianni affiche donc tout au long du film une attitude, « honnête » comme il dit, ce qui l'amènera à sa mort faute de pouvoir faire partager ses sentiments par sa sœur ${ }^{2}$. Sandra est un personnage plus complexe que son frère. Elle est habitée par les fantômes du passé. À son arrivée dans le palais familial, Sandra se dirige dans le jardin où a été érigée la statue du père encore recouverte d'un drap, la caresse et l'enlace pour marquer son amour du père. À ce moment surgit son frère et les deux vont s'enlacer longuement, amoureusement. On ne sait pas si Sandra transfère l'amour passion de son frère vers son père, comme le pense Gianni, ou si c'est l'amour du père qu'elle a d'abord transféré vers son frère pour ensuite revenir vers son père. Visconti fournit différents indices sur les relations incestueuses qu'elle a eues avec son frère ${ }^{3}$. Mais elle n'avoue jamais. Est-elle dans la dénégation, dans le mensonge pour protéger son couple ? C'est très probable mais tout le film laisse planer le doute.

Sandra qui accuse sa mère et son beau-père d'être responsables de la déportation de son père, apparaît comme un personnage tragique pris dans le désir de vengeance, partagée entre l'amour pour son père, pour son mari et pour son frère. Mais son insistance pour accuser sa mère et son beau-père va conduire au drame. La scène finale d'explication entre le frère et la sœur sera suivie du suicide du frère. Le lendemain, Sandra habillée tout en blanc va présider l'inauguration de la statue de son père, en présence de mère et de son beau-père habillés en noir. Le film se termine sur cette scène.

Le frère s'est suicidé parce qu'elle refuse de partager son amour et aussi peut être parce qu'elle nie leur amour de jeunesse. Son mari pris de doute sur les relations incestueuses de sa femme part en espérant qu'elle le rejoindra. C'est 
Sandra qui fabrique l'image de son père pour qui elle manifeste une sorte de passion mais elle le paye au prix fort : la perte d'un être cher, son frère et sans que l'on sache ce qu'il en sera de son mariage. Ce film a été comparé à la tragédie grecque, le personnage de Sandra à celui d'Électre pour signaler que ce ne sont pas les dieux qui punissent les humains mais le destin qui provoque la tragédie.

On saisit la différence entre le film et les fictions romanesques. Si Gianni penche vers l'union fusionnelle comme les héros de Musil, Mann ou Martin du Gard, sa sœur Sandra privilégie la filiation ou plus précisément le rapport au père, à la suite d'un combat contre son amour pour son frère. Mais là encore il ne s'agit pour elle que d'une parenté réduite, la mère et son deuxième mari étant écartés puisque présumés coupables de la mort du père.

\section{Mensonge et filiation dans Mémoires en fuite}

Tout autre est la situation dépeinte dans le téléfilm de François Marthouret (2000) qui commence avec l'inauguration de la statue du Dr Marcel Lemoyne, décédé dans un accident peu après la Deuxième Guerre mondiale. Ce docteur est, dit-on, un héros de la Résistance qui a aidé les juifs au péril de sa vie. La cérémonie se déroule en présence de son fils, Frédéric, médecin lui aussi et de sa fille aînée Jeanne qui restée dans la maison familiale entretient le souvenir glorieux de son père. Cette histoire édifiante sera remise en question dans le film. En réalité le Dr Marcel Lemoyne dénonçait les familles juives à la Gestapo et plaçait leurs biens dans une banque suisse. Le film montre le long et difficile chemin vers la vérité.

À la différence du film de Visconti, il n'est pas question d'inceste entre frère et sœur dans celui de François Marthouret. Jeanne, déjà adulte, s'est occupée de l'éducation de son jeune frère Frédéric sur la base du mensonge concernant leur père. Dans la maison familiale, Jeanne continue à materner et à infantiliser son frère qui se conduit comme un gamin capricieux. A chaque fois qu'il a des doutes sur le passé de son père et vient l'interroger, elle repousse ses suspicions en ajoutant : " j'ai vécu pour toi, j'ai voulu être gaie pour toi, j'ai oublié ton ingratitude ». Mais juste après cette confrontation, elle va accéder à la vérité sans la transmettre à son frère. Jeanne devient une seconde mère pour son frère Frédéric, et tandis qu'il devient le double du père, elle devient la mère de son père, en gommant la part monstrueuse de leur géniteur. Certes elle manipule son frère mais on voit aussi que le mensonge est une manière de manipuler sa propre 
conscience.

Frédéric apprendra la vérité sur son père malgré les dénégations de sa sœur. Il saura ainsi qu'ayant rejoint la Résistance, elle tomba amoureuse du chef de ce réseau qui lui proposa deux solutions : soit dénoncer publiquement son père, soit l'exécuter en déguisant l'assassinat en accident. Elle choisit la deuxième solution.

Dans une des scènes, Jeanne raconte à Frédéric sa dernière rencontre avec son père avant son exécution. Celui-ci avait compris qu'il n'y avait pas d'autre solution. Il annonça à sa fille qu'il avait laissé tout à l'état et lui dit enfin : « «Antigone, mon Antigone »" Frédéric, alors, entoure de ses bras sa sœur, comprenant le sacrifice de sa vie, de son passé de résistante. Se libérant du poids envahissant du père, il va se rapprocher de Jeanne, comprend ses sacrifices et lui témoigne sa compassion.

Alors que dans le film de Visconti, le frère disparaît pour permettre à sa sœur d'assumer le culte du père, dans celui de Marthouret, c'est la remise en question de ce culte qui va replacer au centre la relation frère-sœur. La figure de résistante de Jeanne, meurtrière de son père, empêche le frère de se séparer d'elle, bien qu'elle l'ait élevé dans le mensonge. N'est-il pas devenu un médecin en partie grâce à ce mensonge ? La filiation peut alors s'incarner potentiellement dans le futur, lorsqu'on apprend qu'une chambre va être préparée par Jeanne pour accueillir le fils du frère. Un certain équilibre s'établit entre le frère et la sœur, sinon que pour cette dernière, le mensonge est comme une raison de vivre (ainsi le refus de reconnaître la maîtresse du père). Par ce mensonge, elle espère garder une certaine emprise sur son frère et continuer à être une mère de remplacement.

La relation frère-sœur construit en fin de compte une filiation orientée vers le futur alors que dans le film de Visconti, la sœur s'inscrit dans le rapport au passé, en détruisant sa relation au frère et sans que l'on sache si l'avenir de la filiation par le mariage sera viable. Le mensonge et son dévoilement loin de provoquer des ruptures dans la parenté, construisent les relations, alors que l'inceste est mortifère et fragilise le futur pour ne garder qu'une image du passé. Autrement dit se développent ici deux figures de la relation frère-sœur en rapport avec la filiation : l'une qui est figée sur le culte du passé par la sœur, l'autre qui utilise une figure du passé pour fabriquer le présent et le futur d'une lignée. 
L'amour fou mortifère d'un frère pour sa sœur dans : Bunny Lake a disparu

Dans le film anglais de Otto Preminger ${ }^{5}$, réalisé en 1965, le rapport entre frère et sœur est central, en relation non pas à l'ascendance comme dans les deux films précédents mais à la descendance. Ici une paire frère-sœur arrivant des ÉtatsUnis s'installe à Londres. Le décès de leurs parents est vaguement évoqué. Ni l'un ni l'autre ne sont mariés. Dans un premier temps, il est question de la disparition de la fille de la sœur, conduite pour la première fois à la maternelle et que sa mère venait rechercher dans l'après-midi. Personne à l'école n'a vu la fillette et il n'y a aucune trace de son passage. Tous ses objets et habits ont disparu de l'appartement, ce qui interpelle la police : cette enfant, appelée Bunny Lake, existe-t-elle ? et sa prétendue mère ne l'a-t-elle pas inventée ?

Le film entretient le soupçon et le frère, tout en défendant sa sœur, semble l'accabler. C'est lui qui raconte que sa sœur avait inventé dans son enfance, l'existence d'une fille qu'elle avait aussi appelée Bunny Lake, suggérant ainsi qu'elle reproduit un fantasme de son passé. La sœur assure qu'elle a bien une fille. Pour preuve, le ticket d'un jouet abîmé par sa fille qu'elle a mis en réparation dans un magasin spécialisé. Elle va rechercher le jouet réparé et pendant qu'elle paye, son frère brûle le jouet. Il frappe sa sœur qui s'évanouit et la fait interner dans un hôpital pour délire et pour affabulation. La sœur s'échappe de l'hôpital et revient dans la maison qu'elle a occupée avec son frère, les premiers jours de leur arrivée à Londres où elle retrouve son frère.

La scène finale, magistralement filmée, va totalement changer la donne. Bunny Lake existe bien, le frère l'a enlevée et veut la tuer et l'enterrer avec tous ses jouets et ses habits car il ne supporte plus que sa sœur aime une autre personne que lui. Celle-ci, pour sauver sa fille, va rejouer différentes scènes de l'amour enfantin avec son frère qu'ils ont partagé dans le passé (cache-cache, colinmaillard) pour le détourner de son projet meurtrier. Elle lui demande de la pousser dans une balançoire en attendant l'arrivée de la police qui a trouvé la trace de cette enfant à l'arrivée en Angleterre. La fillette est finalement sauvée et le frère amoureux fou de sa sœur est arrêté et probablement placé dans un centre psychiatrique.

Dans ce film, la filiation est orientée vers le futur et non vers le passé comme dans les deux autres fictions. La relation frère-sœur apparaît comme une menace pour le futur de la filiation. Comme dans le film de Visconti, le frère ne supporte pas que quelqu'un s'interpose entre lui et sa sœur. Différents indices nous sont 
donnés pour indiquer que son projet meurtrier est programmé : il dit avoir envoyé un chèque pour l'inscription de la fillette à l'école or celle-ci ne l'a jamais reçu. Il accuse la police de ne pas faire le nécessaire pour retrouver sa nièce mais c'est lui qui suggère son inexistence. Enfin il avoue dans la dernière scène qu'il a suivi sa sœur quand elle a emmené sa fille à la maternelle, qu'il a profité de son départ pour la chercher et l'enfermer dans le coffre de sa voiture après l'avoir endormie. Alors qu'au début du film, il donne l'impression d'aider sincèrement sa sœur, on découvre par la suite un regard presque halluciné, comme un automate qui exécute sa proie. Le film rappelle dans la scène finale que le frère et la sœur ont eu des relations ambiguës dans leur jeunesse. Mais comme dans le film de Visconti, il faudra briser cet amour passionnel et que le frère disparaisse d'une manière ou d'une autre pour que la sœur s'occupe de sa fille, pour qu'elle fabrique une sorte de lignée féminine exclusive sans frère ni mari.

Les trois fictions romanesques, rédigées durant la première moitié du $\mathrm{xx}^{\mathrm{e}}$ siècle, ne font aucune référence à la Première Guerre mondiale. Aucune interférence d'un événement extérieur tragique ne vient modifier les relations entre les deux protagonistes. Certes dans le roman de Musil, Agathe et Ulrich se retrouvent à l'occasion de la mort de leur père, mais c'est plus une occasion de retrouvailles et le début de leur amour fusionnel qu'un moyen de gérer positivement un rapport au passé, à la filiation. En revanche, deux films Sandra et Mémoire en fuite rappellent différents faits marquants de la Deuxième Guerre mondiale : la délation et la déportation, la collaboration et la Résistance, la spoliation des biens des juifs etc. Ces événements tragiques traversent le temps pour venir hanter les personnages longtemps après la fin de la guerre. Le souvenir comme les blessures du passé sont ravivés, vont évoluer ou trouver leur épilogue. La guerre perturbe les relations de parenté et celles-ci à leur tour vont modifier la mémoire de la guerre. La mémoire du passé est donc bien l'œuvre du présent. Les deux fictions fonctionnent en miroir car il s'agit dans les deux cas de gérer la disparition d'un père longtemps après. Les deux filles s'acharnent à défendre sa mémoire : Sandra est très probablement à l'origine de l'érection de la statue du son père, victime selon elle d'une dénonciation ; pour sa part, Jeanne sait que son père était un collaborateur et pourtant elle s'est obstinée pour qu'on lui élève une statue cinquante ans plus tard. De même, dans les deux films, la mère n'est jamais associée à la fabrication de cette image du père. Dans Sandra, la mère pianiste renommée est accusée par sa fille d'être responsable de la mort du père. Dans Mémoire en fuite, la dépression de la mère est évoquée, et sa fille n'a 
aucunement cherché à l'associer à l'hommage rendu au père.

Le troisième film, Bunny Lake a disparu est différent, car il met en scène l'arrivée de la paire frère-sœur à Londres, leur installation dans une ville où ils travaillent. Et il est important de signaler que le frère n'a pas cherché à tuer la fille de sa sœur dans leur pays d'origine, les États-Unis, mais dans celui où ils sont des étrangers. Il s'agit donc d'une situation inverse à celle des deux autres films qui se déroulent dans le lieu d'origine de la paire frère-sœur et même dans leur maison de naissance. À cela, il faut ajouter comme on l'a dit plus haut que le trio dont il est question dans le film de Preminger n'est pas celui de la paire de germains et du père, mais du frère, de la sœur et de la fille de cette dernière.

Les différentes fictions étudiées dans cet article mettent en scène soit l'identification du frère et de la sœur, soit leur séparation avec une asymétrie entre les deux termes de la relation. Le désir d'inceste, l'amour fusionnel sont présentés comme mortifères du point de vue de la parenté (Musil, Martin du Gard). Le film de Visconti peut être considéré comme un cas intermédiaire : le frère désire une relation amoureuse passionnelle avec sa sœur alors que celle-ci s'inscrit dans la filiation en privilégiant la relation à son père décédé. Il en est de même pour le film de Preminger si ce n'est que la filiation est orientée vers le futur. En revanche, dans le film de Marthouret, le mensonge et son dévoilement dans la relation entre germains de sexe opposé peuvent construire de manière paradoxale une lignée agnatique sur trois générations.

A propos de l'asymétrie, il serait erroné de considérer que c'est le frère qui prédomine sur sa sœur, qu'il occupe la position de l'aîné par rapport à sa cadette. $\mathrm{Au}$ contraire, c'est la sœur qui mène l'action alors que le frère bénéficie des effets de cette action ou en subit les effets. Sandra consacre la mémoire du père déporté en rejetant l'amour du frère qui se tue faute de pouvoir le faire partager. Jeanne l'aînée a tué littéralement son père et fabriqué son frère à l'image de ce père. La mère de Bunny Lake n'a qu'un objectif, sauver sa fille des griffes meurtrières de son frère. Dans ces différents exemple, la lignée (agnatique ou féminine) est construite par l'action de la sœur ${ }^{6}$.

Parler de dominance à propos de la relation entre frère et sœur ne me paraît pas pertinent dans les différents cas étudiés car c'est réduire les relations sociales à un affrontement entre des intérêts opposés. Il s'agit plutôt de voir comment se définissent les formes de relations entre cette paire de germains de sexe opposé : entre identité et différenciation, entre mariage et consanguinité, entre négation 
ou construction de la filiation.

Dans un article sur le vocabulaire de la parenté en Inde du nord, Louis Dumont [1962] compare les trois types de parenté en en soulignant les paramètres principaux : complémentarité entre consanguinité et affinité dans la parenté dravidienne de l'Inde du sud, prédominance de la relation frère-sœur dans la parenté hindi du nord de l'Inde et enfin distinction entre ligne directe et lignes collatérales à l'intérieur de la consanguinité dans le vocabulaire français. Il me semble qu'il faut modifier la proposition de Louis Dumont pour rendre ces cas concrets comparables. En effet dans les trois types de parenté auxquels il fait référence, la relation frère-sœur constitue le chaînon opératoire dans la construction diachronique de la parenté mais de manière différente. Dans la parenté dravidienne de l'Inde du sud, le frère donne sa sœur en mariage et épouse la sœur d'un autre homme, l'échange matrimonial se répétant sur plusieurs générations. En Inde du nord, la sœur mariée revient en tant qu'officiante chez son frère pour le mariage des enfants de celui-ci. À la génération suivante, c'est sa nièce, la fille mariée de son frère qui prendra sa place. Plus généralement, en Inde, la relation frère-sœur se définit, génération après génération, dans la répétition de l'alliance de mariage ou de l'action rituelle.

Dans nos fictions cinématographiques, la relation frère-sœur opère dans la construction sociale de la filiation, soit en séparant les deux germains de sexe opposé (Sandra et Bunny Lake a disparu), soit au contraire en solidifiant leur unité (Mémoires en fuite). Si en Inde la relation frère-sœur est tournée vers le futur, dans les fictions, l'obsession est de construire la figure ancestrale, notamment avec l'érection de statues, ou au contraire de tuer le père, tout en perpétuant sa figure, pour construire, éventuellement, l'avenir ou encore arrêter le frère qui menace de tuer la fille de sa sœur. Dans le trio que constitue frère, sœur et père ou frère, sœur et fille, l'un des termes doit être éliminé pour que les deux autres puissent s'affirmer. Les différents cas examinés dans cet essai montrent que la relation frère-sœur est un opérateur important de la parenté. Ils n'épuisent pas toutes les potentialités de cette relation que l'analyse d'autres exemples viendra enrichir et complexifier.

\section{Notes}

1. Pour une présentation générale de cette question, voir le travail de l'historien Didier Lett 
[2009], celui du sociologue Eric Widmer [1995]. Une mention particulière doit être faite à une analyse de Colette Fellous [1992] qui mêle des souvenirs personnels aux références littéraires où sont évoquées les relations étroites entre différents auteurs (Châteaubriand, Bal- zac, Stendhal, etc.) et leur sœur.

2. Gianni qui a pris du poison et qui est en train de mourir, a écrit une lettre à sa sœur pour l'implorer de ne pas l'abandonner alors qu'il est tombé dans l'abîme. La caméra filme longuement sa mort dans d'affreuses douleurs.

3. Dans une longue scène, Sandra raconte à son mari son enfance et ses relations avec son frère. Malgré la volonté du beau-père de les séparer, ils se retrouvaient, se rapprochaient en cachant leur complicité, en laissant des notes dans différents endroits pour se fixer des ren- dez-vous loin des regards.

4. Le père de Jeanne se réfère, très probablement, non pas à la pièce de théâtre de Sophocle mais à celle de Jean Anouilh qui a été jouée une première fois en 1944 durant l'occupation allemande et dans laquelle Antigone apparaît comme la figure de toutes les révoltes et notamment de la Résistance.

5.Je voudrais remercier Charles Malamoud qui m'a signalé ce film et avec lequel j'ai eu plusieurs discussions intéressantes à ce sujet.

6. Pour une approche différente mettant l'accent plutôt sur la prééminence du frère sur la sœur, voir Françoise Héritier [2013].

\section{Références bibliographiques}

Dumont Louis, 1962, "Le vocabulaire de parenté dans l'Inde du nord », L'Homme II, (2:5-48)

Dumont Louis, 1975. Dravidien et Kariera, Paris / La Haye - Mouton

Fellous Colette, 1992, Frères et sœurs, Paris, Julliard 
Héritier Françoise, 2013, Le rapport frère/sœur, pierre de touche de la parenté. Conférence de Eugène Fleischman. Nanterre, Société d'ethnologie.

Jamous Raymond, 1991, La relation frère-sæur. Parenté et rites chez les Meo de l'Inde du nord, Paris, Editions de l'ehess.

Lett Didier, 2009, Frères et sœurs. Histoire d'un lien, Paris, Petite Bibliothèque Payot.

Lévi-Strauss Claude, 1967 [1949], Les structures élémentaires de la parenté, Paris, Presses universitaires de France.

Malamoud Charles, 2002, "Frères et sœurs dans l'Inde Brahmanique », Le jumeau solaire, Paris, Le Seuil : 35-52.

Widmer Eric, 1995, D'Abel et de Caïn. Les relations fraternelles à l'adolescence, Thèse de sociologie de l'Université de Genève.

\section{Romans cités}

Mann Thomas, 1905, Sang réservé suivi de Désordre, Paris, Livre de poche.

Martin du Gard Roger, 1949, Confidence africaine. Paris, Gallimard.

Musil Robert, 1932, L'Homme sans qualités, tome II.

\section{Films cités}

Marthouret François, 2000, Mémoires en fuite (téléfilm).

Preminger Otto, 1965, Bunny Lake a disparu (Bunny Lake is missing).

Visconti Luchino, 1965, Sandra (Vaghe stelle dell'Orsa). 